\title{
Differences in Karyotype and Fluorochrome Banding Patterns among Variations of Trichosanthes cucumerina with Different Fruit Size
}

\author{
Biplab Kumar Bhowmick ${ }^{1,2}$ and Sumita Jha2* \\ ${ }^{1}$ Department of Botany, Scottish Church College, 1 and 3, Urquhart Square, Kolkata-700006, India \\ ${ }^{2}$ CAS, Department of Botany, University of Calcutta, 35, Ballygunge Circular Road, Kolkata-700019, India \\ Received December 31, 2018; accepted April 20, 2019
}

\begin{abstract}
Summary Snake gourd is an agriculturally important cucurbit vegetable recognized presently as the cultivar 'Anguina' of Trichosanthes cucumerina ssp. cucumerina. The wild type plant occurs naturally that produces small fruits (TCSF) and thus can be distinguished from the cultivar with large elongated fruits (TCLF). Presently, chromosomal features were revealed by modern cytogenetic methods to characterize the two types of plants. Chromosome preparations were standardized by an enzymatic maceration and air-drying method (EMA). The cultivars had considerably different karyotypes than the TCSF plants in spite of the same chromosome numbers $(2 n=22)$. EMA-DAPI based meiotic cell preparations reconfirmed gametic chromosome number $(n=11)$ and showed regular chromosome behavior in both plant types. Karyomorphometric study with 14 inter- and intra-chromosomal symmetry/asymmetry indices advocated higher asymmetry in the karyotype of TCLF. The fluorochrome banding pattern of somatic chromosomes revealed the differential distribution of CMA and DAPI bands in the types of plants. TCSF plants were characterized by the presence of CMA bands in two pairs of chromosomes with secondary constrictions while DAPI bands were found in all chromosomes of the complements. On the contrary, DAPI bands were completely absent in TCLF while distal CMA bands were scored in two pairs of chromosomes with secondary constrictions and one pair of other chromosomes. CMA-DAPI banding patterns and karyomorphometric parameters had a significant impact on the evaluation of karyotype relations. The plants of TCSF and TCLF formed distinct groups and clusters in PCA plot and UPGMA phenogram, respectively. The variation in karyoevolutionary status and fluorochrome banding pattern between TCSF and TCLF plants could be utilized for further cytotaxonomic and phylogenetic revisions.
\end{abstract}

Key words EMA-Giemsa, CMA, DAPI, Snake gourd, Trichosanthes cucumerina L., Karyotype asymmetry, Meiosis.

The family Cucurbitaceae has been esteemed since ancient times for being the world's second large vegetable producing family extensively cultivated in tropical countries. Like many other cucurbit crops, snake gourd is one of the widely consumed vegetables in India which was known as a variety of Trichosanthes cucumerina L. [viz. T. cucumerina L. var. anguina (L.) Haines] for a long time (Haines 1922). T. cucumerina is a native species of Trichosanthes in India (Renner and Pandey 2013) belonging to the tribe Sicyoeae of Cucurbitaceae (Schaefer and Renner 2011). T. cucumerina produces small ovoid fruits while the snake gourds are elongated and sometimes twisted fruits used as a vegetable when unripe. The distinct difference in fruit morphology was the reason why $T$. cucumerina and $T$. anguina were origi-

\footnotetext{
*Corresponding author, e-mail: sumitajha.cu@gmail.com DOI: $10.1508 /$ cytologia. 84.237

This paper is dedicated to the memory of our teacher and renowned Plant Cytogeneticist Professor Arun Kumar Sharma (31st December 1924-6th July 2017), who worked tirelessly to improve plant science teaching and research in the country.
}

nally entitled as different species (Linnaeus 1753) before the inclusion of snake gourd as a variety of T. cucumerina (Haines 1922). Chakravarty (1959) re-established the rank of two distinct species based on fruit character. Five species of Trichosanthes were later assembled into T. cucumerina group (Jeffrey 1980a, b, Chakravarty 1982) including T. cucumerina and T. anguina owing to variable and overlapping morphological characters. Molecular phylogenetic studies supported the assignment of the vegetable snake gourd as a variant form of T. cucumerina (Ali and Al-Hemaid 2010, Cooper and de Boer 2011). Of late, T. cucumerina group has been worked out in detail and includes infraspecific ranks based on reliable morphological characters (Pradheep et al. 2015). T. cucumerina has three subspecies of which T. cucumerina ssp. cucumerina is the one distinguished from its cultivar again by one attribute i.e., elongated fruits (the snake gourds). Hence snake gourd has been regarded as the cultivar T. cucumerina ssp. cucumerina Anguina. (L.) K. Pradheep, D.R. Pani \& K.C. Bhatt, cult. nov. of the wild T. cucumerina ssp. cucumerina (Pradheep et al. 
2015) and not a variant form or variety.

Genomic or cytogenetic analysis of the species is missing although snake gourd ('Anguina') cultivar and its wild type plant have huge distribution and rich germplasm in India. The recent taxonomic revision is not substantiated by the genomic or chromosomal study. A cytogenetic investigation also has a major role in breeding programs for many important crops like wheat or maize (Albert et al. 2010, Icso et al. 2014). The dearth of cytogenetic information encouraged us to study the basic chromosomal features of T. cucumerina. So far, chromosome numbers in $T$. cucumerina $(2 n=22, n=11)$ were reported (McKay 1931, Nakajima 1936, Sen and Datta 1975, Singh and Roy 1975, Datta and Basu 1978, Sarker et al. 1987, Beevy and Kuriachan 1996) while conventional chromosome staining was followed to construct karyotypes in T. cucumerina and T. anguina (Sarker et al. 1987, Alam et al. 2012, 2018). Cucurbit species have small chromosomes and dense cytoplasmic content (Bhowmick and Jha 2015, Ghosh et al. 2018) that might obstruct thorough analysis via conventional methods. The EMA (Fukui 1996) helps in the elimination of cell wall and cytoplasm required for the scattering of chromosomes (Bhowmick et al. 2012). We have taken up this method for mitotic and meiotic chromosome study in $T$. cucumerina. This was accompanied by the analysis of fluorochrome banding pattern in the chromosomes using chromomycin $\mathrm{A}_{3}$ (CMA) and 4',6-diamidino-2-phenylindole (DAPI). We discuss how cytogenetic information can be used to understand the karyoevoutionary status and possible variation between wild plants of T. cucumerina and the snake gourd cultivar which is a prerequisite for further cytotaxonomical analyses.

\section{Materials and methods}

\section{Plant materials}

For the convenience of interpretation, T. cucumerina ssp. cucumerina was designated as the small fruit producing plant (TCSF, fruit size $4-5 \mathrm{~cm}$, Fig. 1a) and Trichosanthes cucumerina ssp. cucumerina 'Anguina' (L.) was designated as large fruit producing cultivar i.e., snake gourd (TCLF, fruit elongated, $15-30 \mathrm{~cm}$ long or even more, Fig. 1b, c). TCSF plants grow naturally in various regions of India. Entire TCSF plants were collected from Ballygunge, Kolkata, and University of Agricultural Sciences, Bangalore and seeds were collected from NBPGR, Thrissur (Table 1). Seeds of TCLF were collected from Bongaon, West Bengal, nurseries of Bhagalpur, Bihar and Adiganahalli, Bengaluru (Table 1). Seedlings were grown and plants were maintained in the Experimental Garden of University of Calcutta.

\section{Chromosome preparation}

Somatic chromosomes from root tips were derived from germinating seedlings and underground rhizomes.

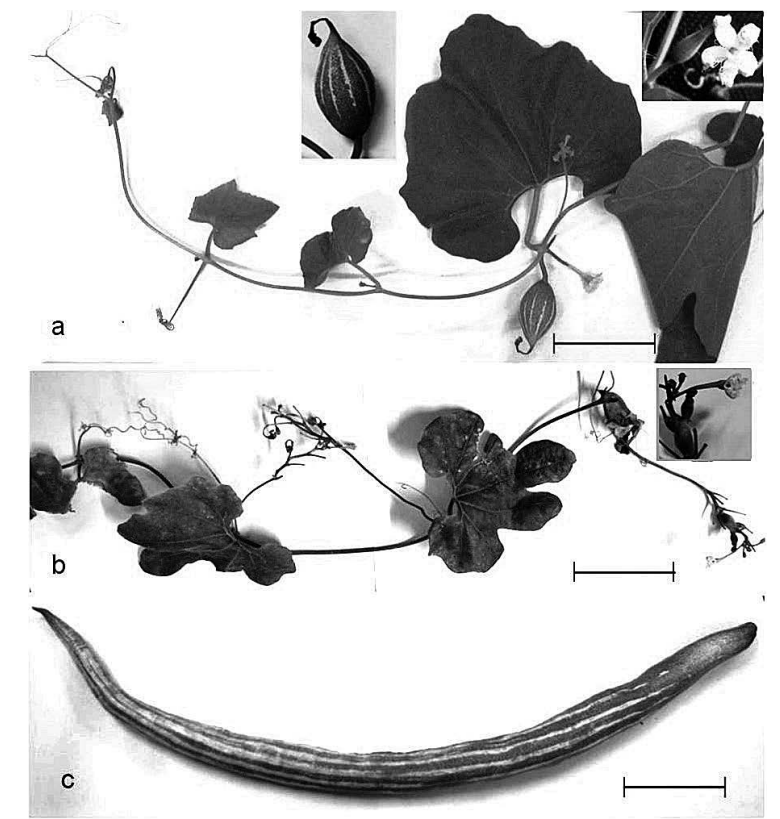

Fig. 1. TCSF plant with enlarged flower and fruit in insets (a). TCLF plant (cultivar) with an enlarged flower in the inset (b) and the fruit of TCLF (snake gourd) cultivar (c). Scale bar $=5 \mathrm{~cm}$.

Table 1. Collection details of Trichosanthes cucumerina.

\begin{tabular}{clc}
\hline \hline Type of fruits & \multicolumn{1}{c}{ Population } & $\begin{array}{c}\text { Abbreviation of } \\
\text { population }\end{array}$ \\
\hline \multirow{2}{*}{ TCSF } & Ballygunge, Kolkata, West Bengal & KOL \\
& NBPGR, Thrissur, Kerala & NBP \\
& University of Agricultural Sciences & BEN \\
& GKVK, Bengaluru, Karnataka & \\
\hline \multirow{2}{*}{ TCLF } & Bongaon, West Bengal & BON \\
& Bhagalpur, Sabour Diara, Bihar & BHA \\
& Adiganahalli, North Bengaluru, & ADI \\
& Karnataka & \\
\hline
\end{tabular}

Healthy roots $(0.5-1 \mathrm{~cm}$ in length) were pretreated in $2 \mathrm{mM}$ hydroxyquinoline at $15^{\circ} \mathrm{C}$ for $4 \mathrm{~h}$ and fixed in freshly prepared aceto-methanol $(1: 3)$. Root tips were subjected to EMA (Fukui 1996) with useful modifications. Root tips were digested in a cocktail of $1 \%$ cellulase Onuzuka RS (Yakult Pharmaceutical Ind. Co., Ltd., Japan), 0.15\% pectolyase Y-23 (Yakult Pharmaceutical Ind. Co., Ltd., Japan), 0.75\% macerozyme R-10 (Kyowa Chemical Products Co., Ltd., Japan) and $1 \mathrm{mM}$ EDTA ( $\mathrm{pH} \mathrm{4.2)}$ for $42 \mathrm{~min}$ at $37^{\circ} \mathrm{C}$ followed by maceration on clean glass slides in a drop of the fixative. The air-dried slides were stained with 2\% Giemsa [Giemsa solution (Merck): 1/15M phosphate buffer: distilled water $=2: 50: 48]$ for $20 \mathrm{~min}$. Metaphase plates were observed under a Leitz GMBH microscope attached with a ProgRes CT5 Jenoptik D07739 camera and a ProgRes CapturePro ver 2.8.8 software was used for capturing photographs and taking measurements [long arm length (1), short arm length (s), chromosome length (CL), total diploid chromosome length (TCL)]. For karyotype 
analysis, chromosomes were classified on the basis of centromeric indices $[\mathrm{Ci}=(\mathrm{s} / \mathrm{CL}) \times 100]$ according to Levan et al. (1964) and designated as metacentric (M) (Ci>47.5-50.0), nearly metacentric (m) $(\mathrm{Ci}>37.5-47.5)$, submetacentric $(\mathrm{sm})(\mathrm{Ci}>25.0-37.5)$ and subtelocentric (st) $(\mathrm{Ci}>12.5-25.0)$.

\section{Analysis of karyomorphometric parameters}

At least five metaphase plates from five individual plants of TCSF and those of TCLF from each population had been selected for karyotype preparation and estimation of the degree of symmetry/asymmetry by 14 different parameters. The chromosomes were arranged in decreasing order of length for presenting the karyotype. Inter- and intra-chromosomal asymmetry/symmetry in the karyotypes were evaluated after calculating values of indices viz. total form percentage ( $\mathrm{TF} \%$ ) (Huziwara 1962), intrachromosomal asymmetry index (A1) and interchromosomal asymmetry index (A2) (Zarco 1986), coefficients of variation of chromosome length $\left(\mathrm{CV}_{\mathrm{CL}}\right)$ and arm ratio $\left(\mathrm{CV}_{\mathrm{r}}\right)$ (Paszko 2006), index of karyotype symmetry (Syi) (Greilhuber and Speta 1976), asymmetry index of karyotype (AsK\%) (Arano 1963), degree of karyotype asymmetry (A) (Watanabe et al. 1999), mean centromeric index $\left(\mathrm{X}_{\mathrm{CI}}\right)$ (Seijo and Fernández 2003), coefficient of variation of centromeric index $\left(\mathrm{CV}_{\mathrm{CI}}\right)$ (Paszko 2006), symmetry index (S\%) (Liang and Chen 2015), dispersion index (DI) (Lavania and Srivastava 1992), asymmetry index (AI) (Paszko 2006) and mean centromeric asymmetry $\left(\mathrm{M}_{\mathrm{CA}}\right)$ (Peruzzi and Eroğlu 2013).

\section{Fluorochrome staining of somatic metaphase chromo- somes}

The Giemsa stained metaphase chromosome slides were marked under a fluorescent microscope Zeiss Axioscop 2 followed by destaining in $70 \%$ methanol for $45 \mathrm{~min}$ and air drying for sequential DAPI and CMA staining according to the protocol (Bhowmick et al. 2012). Slides were incubated in McIlvaine buffer $\left(0.1 \mathrm{M}\right.$ citric acid, $\left.0.2 \mathrm{M} \mathrm{Na}_{2} \mathrm{HPO}_{4}, \mathrm{pH} 7.0\right)$ for $15 \mathrm{~min}$ and stained with $20 \mu \mathrm{gmL}^{-1}$ DAPI for $10 \mathrm{~min}$ in the dark phase, washed in the buffer, blow-dried, mounted in non-fluorescent glycerol and observed under the fluorescence microscope with a UV filter cassette. After observation with DAPI, the slides were de-stained in the fixative for $30 \mathrm{~min}$, air-dried and incubated in the buffer with $5 \mathrm{mM} \mathrm{MgSO}_{4}$ for $10 \mathrm{~min}$. Next, the slides were stained with $0.1 \mathrm{mg} \mathrm{mL}^{-1} \mathrm{CMA}$ for $10-15 \mathrm{~min}$ in the dark phase, washed with the buffer with $5 \mathrm{mM}$ $\mathrm{MgSO}_{4}$, blow-dried and finally mounted in glycerol. Observations were taken under a BV filter cassette in the fluorescence microscope. Images were captured with the camera ProgRes MF scan Jenoptik D07739 attached to the fluorescence microscope using the software ProgRes CapturePro 2.8.8.
Preparation of meiotic chromosomes by EMA-DAPI technique

Dense cytoplasmic content in the PMCs was eliminated by EMA-DAPI staining (Bhowmick and Jha 2015) with minor modifications. The male flower buds of $0.5 \mathrm{~cm}$ length were collected at around $10 \mathrm{am}$ and macerated in the enzyme mixture for $3-4 \mathrm{~min}$ at $37^{\circ} \mathrm{C}$. Macerated anthers were pipetted out in a clean glass slide and PMCs were spread in the fixative and air-dried for $1-3 \mathrm{~h}$. The slides were kept in the buffer for $12 \mathrm{~min}$. Slides were then stained with $20 \mu \mathrm{g} \mathrm{mL}^{-1}$ DAPI solution for $20 \mathrm{~min}$, washed in the buffer and finally mounted in glycerol. The observation was taken under the UV filter in the fluorescence microscope and images were captured using the camera.

\section{Evaluation of chromosomal relationships by statistical analyses}

One way analysis of variance (ANOVA) and Duncan's multiple range test (DMRT) was conducted for chromosome morphometric data and karyotype study considering triplicate values of the TCSF and TCLF plants using IBM SPSS Statistics 16.0. Quantitative parameters of chromosome measurements and karyomorphometric values were used to assess significant differences ( $p \leq 0.05$ at $95 \%$ confidence level) between TCSF and TCLF if any. A cluster analysis with nine numerical karyotype parameters $\left(\mathrm{CV}_{\mathrm{CL}}, \mathrm{CV}_{\mathrm{r}}, \mathrm{AsK} \%, \mathrm{CV}_{\mathrm{CI}}, \mathrm{M}_{\mathrm{CA}}\right.$, DI, AI, number of chromosomes with DAPI and CMA signals) was conducted to normalize data matrix and produce an unweighted pair group method with arithmetic mean (UPGMA) dendrogram based on average Euclidean distance using Infostat 2017/d software. Apart from this, we also focused on the principal component analysis (PCA) with the above mentioned karyomorphometric parameters using the same software since PCA is a useful statistical measure for multivariate analysis (McVean 2009, Marghali et al. 2014).

\section{Results}

\section{Karyotype features based on EMA-Giemsa staining}

Standardization of pretreatment conditions combined with EMA-Giemsa method resulted in scattered metaphase plates with clear chromosomes against a cytoplasm free background suitable for karyotype analysis in the plants (Fig. 2). The number of chromosomes in somatic cells was $2 n=22$ in both TCSF (Fig. $2 \mathrm{a}-\mathrm{c}$ ) and TCLF (Fig. 2f-h). Chromosome length in a complement ranged from $2.26-4.99 \mu \mathrm{m}$ in TCSF (Table 2). The karyotype was composed of 18 nearly metacentric and four nucleolar chromosomes. One of the nucleolar pairs was submetacentric with a secondary constriction on the short arm and the other pair was nearly metacentric with a secondary constriction on the short arm (Fig. 2a-c). Plants of TCSF were found to have consistency in the 


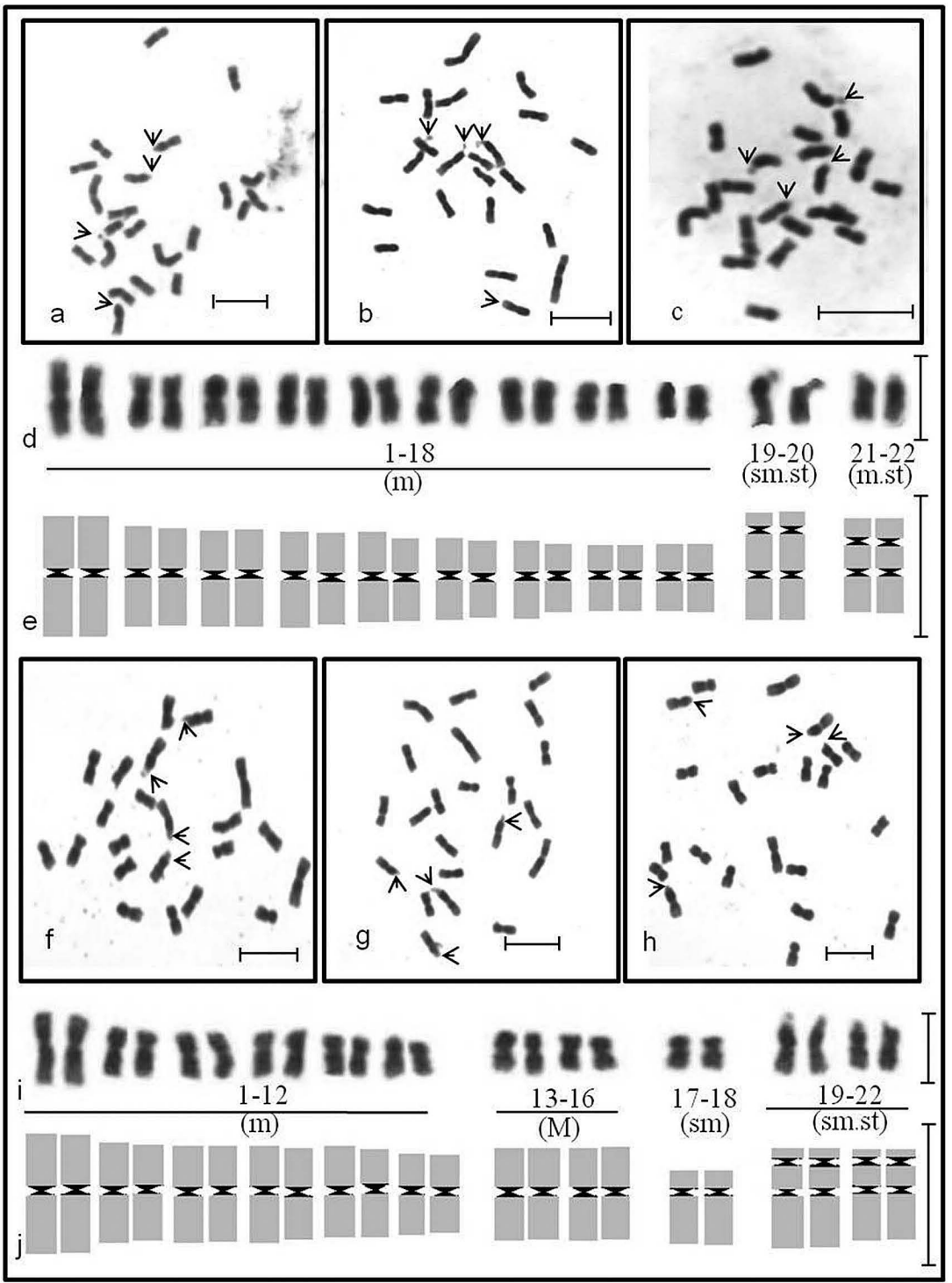

Fig. 2. EMA-Giemsa preparations of somatic metaphase chromosomes $(2 n=22)$ of TCSF plants collected from populations KOL (a), NBP (b) and BEN (c). d-e Karyotype and corresponding ideogram of TCSF. $\mathrm{f}-\mathrm{h}$ EMAGiemsa preparations of somatic metaphase chromosomes $(2 n=22)$ of TCLF plants collected from populations BON (f), BHA (g) and ADI (h). $\mathrm{i}-\mathrm{j}$ Karyotype and corresponding ideogram of TCLF. Chromosomes with nucleolar constrictions are indicated by arrows. For abbreviation of populations, see Table 1 . Scale bar $=5 \mu \mathrm{m}$.

Table 2. Chromosome number, karyotype data and CMA-DAPI banding pattern in T. cucumerina.

\begin{tabular}{|c|c|c|c|c|c|c|c|c|c|}
\hline \multirow{2}{*}{$\begin{array}{l}\text { Type of } \\
\text { fruits }\end{array}$} & \multirow{2}{*}{ Population } & \multicolumn{2}{|c|}{$\begin{array}{c}\text { Chromosome } \\
\text { number }\end{array}$} & \multirow{2}{*}{$\mathrm{TCL}^{*}(\mu \mathrm{m})$} & \multirow{2}{*}{$\operatorname{CSR}^{* *}(\mu \mathrm{m})$} & \multirow{2}{*}{ Karyotype } & \multirow{2}{*}{$\begin{array}{c}\text { Chromosomes } \\
\text { with CMA } \\
\text { bands }\end{array}$} & \multirow{2}{*}{$\begin{array}{l}\text { Chromosomes } \\
\text { with DAPI bands }\end{array}$} & \multirow{2}{*}{$\begin{array}{l}\text { Fluorochrome } \\
\text { banding pattern }\end{array}$} \\
\hline & & $2 n$ & $n$ & & & & & & \\
\hline \multirow{3}{*}{ TCSF } & KOL & 22 & 11 & $76.95 \pm 1.22$ & $2.26-4.99$ & $18 \mathrm{~m}+2 \mathrm{sm}^{\mathrm{st}}+2 \mathrm{~m}^{\mathrm{st}}$ & 4 & 22 & $12 \mathrm{~A}+6 \mathrm{~B}+4 \mathrm{C}$ \\
\hline & NBP & 22 & 11 & $74.21 \pm 1.05$ & $2.29-4.78$ & $18 m+2 s m^{s t}+2 m^{s t}$ & 4 & 22 & $12 \mathrm{~A}+6 \mathrm{~B}+4 \mathrm{C}$ \\
\hline & BEN & 22 & 11 & $75.97 \pm 2.41$ & $2.32-4.68$ & $18 \mathrm{~m}+2 \mathrm{sm}^{\mathrm{st}}+2 \mathrm{~m}^{\mathrm{st}}$ & 4 & 22 & $12 \mathrm{~A}+6 \mathrm{~B}+4 \mathrm{C}$ \\
\hline \multirow[t]{3}{*}{ TCLF } & BON & 22 & 11 & $75.49 \pm 2.85$ & $2.77-5.01$ & $12 \mathrm{~m}+4 \mathrm{M}+2 \mathrm{sm}+4 \mathrm{sm}^{\mathrm{st}}$ & 6 & 0 & $2 \mathrm{D}+2 \mathrm{E}+2 \mathrm{~F}+6 \mathrm{G}$ \\
\hline & BHA & 22 & 11 & $76.11 \pm 0.57$ & $2.80-4.76$ & $12 \mathrm{~m}+4 \mathrm{M}+2 \mathrm{sm}+4 \mathrm{sm}^{\mathrm{st}}$ & 6 & 0 & $2 \mathrm{D}+2 \mathrm{E}+2 \mathrm{~F}+6 \mathrm{G}$ \\
\hline & ADI & 22 & 11 & $75.20 \pm 1.88$ & $2.0-4.98$ & $12 \mathrm{~m}+4 \mathrm{M}+2 \mathrm{sm}+4 \mathrm{sm}^{\mathrm{st}}$ & 6 & 0 & $2 \mathrm{D}+2 \mathrm{E}+2 \mathrm{~F}+6 \mathrm{G}$ \\
\hline
\end{tabular}

*TCL: Total diploid chromosome length, ${ }^{* * C S R}$ : chromosome size range

morphology of the chromosomes. The nucleolar pairs of chromosomes were the longest in the complement. One of the nearly metacentric pair of chromosomes had a slightly longer length than the other nearly metacentric chromosomes in all TCSF individuals (Fig. 2d). The length of this pair ranged from 4.72 to $4.67 \mu \mathrm{m}$ in the plants. The total diploid chromosome length of TCSF was $75.71 \pm 1.38 \mu \mathrm{m}$ (Table 2) and modal karyotype was determined as $18 \mathrm{~m}+2 \mathrm{sm}^{\text {st }}+2 \mathrm{~m}^{\text {st }}$ (Fig. 2d, e, Table 2).

The TCLF plants had six nearly metacentric pairs, two metacentric pairs, one submetacentric pair and two nucleolar pairs of chromosomes (Fig. 2f-j). The pairs of nucleolar chromosomes were the longest and had secondary constrictions at the submedian and subter- 
minal regions in all plants (Fig. $2 \mathrm{i}-\mathrm{j}$ ). Chromosome size ranged from 2.77-5.01 $\mu \mathrm{m}$ in the TCLF plants (Table 2). Among the chromosomes without secondary constriction, one nearly metacentric pair of chromosomes was larger than the others in TCLF plants. The length of this pair was varying between 4.92 to $4.80 \mu \mathrm{m}$. The total diploid chromosome length in TCLF was $76.26 \pm 0.46 \mu \mathrm{m}$ (Table 2) and modal karyotype was found to be $12 \mathrm{~m}+4 \mathrm{M}+2 \mathrm{sm}+4 \mathrm{sm}^{\text {st }}$ (Fig. 2i, j, Table 2).

Comparative karyomorphometric study in TCSF and TCLF plants

Among the 14 parameters taken for the present analysis, the increase in values of $\mathrm{A} 2, \mathrm{CV}_{\mathrm{CL}}$, and $\mathrm{CV}_{\mathrm{r}}$ in TCLF plants provided support for higher inter-chromosomal asymmetry in the karyotype (Table 3 ). Again, a higher level of asymmetry in TCLF compared to TCSF was supported by an increase in the intra-chromosomal asymmetry indices like Ask\% and $\mathrm{CV}_{\mathrm{CI}}$ (Table 3). Remarkably, the intra-chromosomal asymmetry indices showed greater asymmetry in TCLF (Table 3) than those of TCSF. Some of the intra-chromosomal parameters are inversely related to asymmetry and they showed a distinctly lower value in TCLF (A1, TF\%, Table 3). Also, AI value suggested a predisposition of asymmetry in the karyotype of TCLF plants. Cumulatively, 10 out of 14 karyomorphometric parameters depicted an asymmetric nature in the karyotypes of TCLF compared to TCSF plants.

Fluorochrome banding pattern in somatic chromosomes

Differential distribution of CMA and DAPI bands was clear in the two types of plants under study. In TCSF plants, chromosomal DAPI and CMA bands were observed (Fig. 3a-f) and DAPI bands were found in all somatic chromosomes (Fig. 3b, e). A clear examination of DAPI banding revealed six pairs of nearly metacentric chromosomes with DAPI bands in the distal parts of both the arms and three other pairs with distal DAPI bands in the long arm (Fig. 3a, b). CMA staining revealed positive bands in the distal parts of four nucleolar chromosomes (Fig. 3c, d, f). CMA bands were found to be DAPI negative while distal part of the long arm of the same chromosomes was found to have DAPI bands (Fig. $3 \mathrm{a}-\mathrm{c}, \mathrm{e}-\mathrm{f})$.

In the TCLF plants, there were six distinct CMA bands in three pairs of chromosomes (Fig. 3j-m). One of the nucleolar chromosome pair showed distal CMA bands and two CMA spots just below the secondary constriction (Fig. 3i-m). The other chromosome pair displayed a single distal CMA band in the short arm (Fig. $3 \mathrm{i}-\mathrm{j}, \mathrm{k}-\mathrm{m})$. Another distal CMA band was detected in a nearly metacentric pair of chromosomes (Fig. 3i-m). Chromosomes displayed uniform staining with DAPI indicating an absence of any DAPI bands in TCLF plants (Fig. 3n-o).

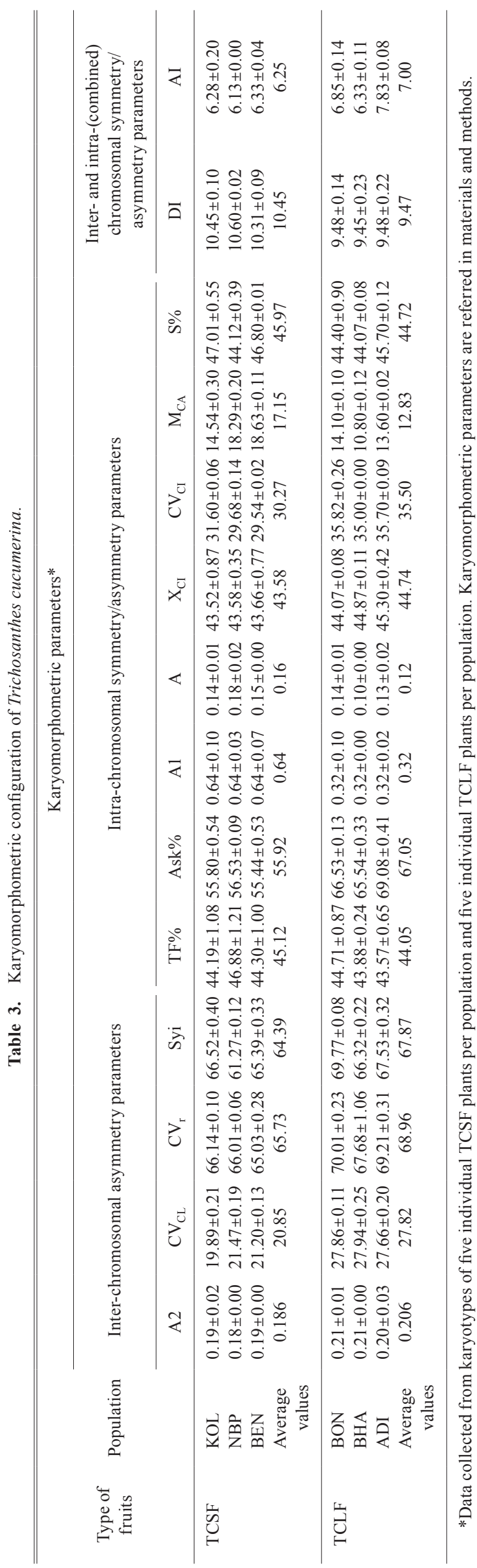




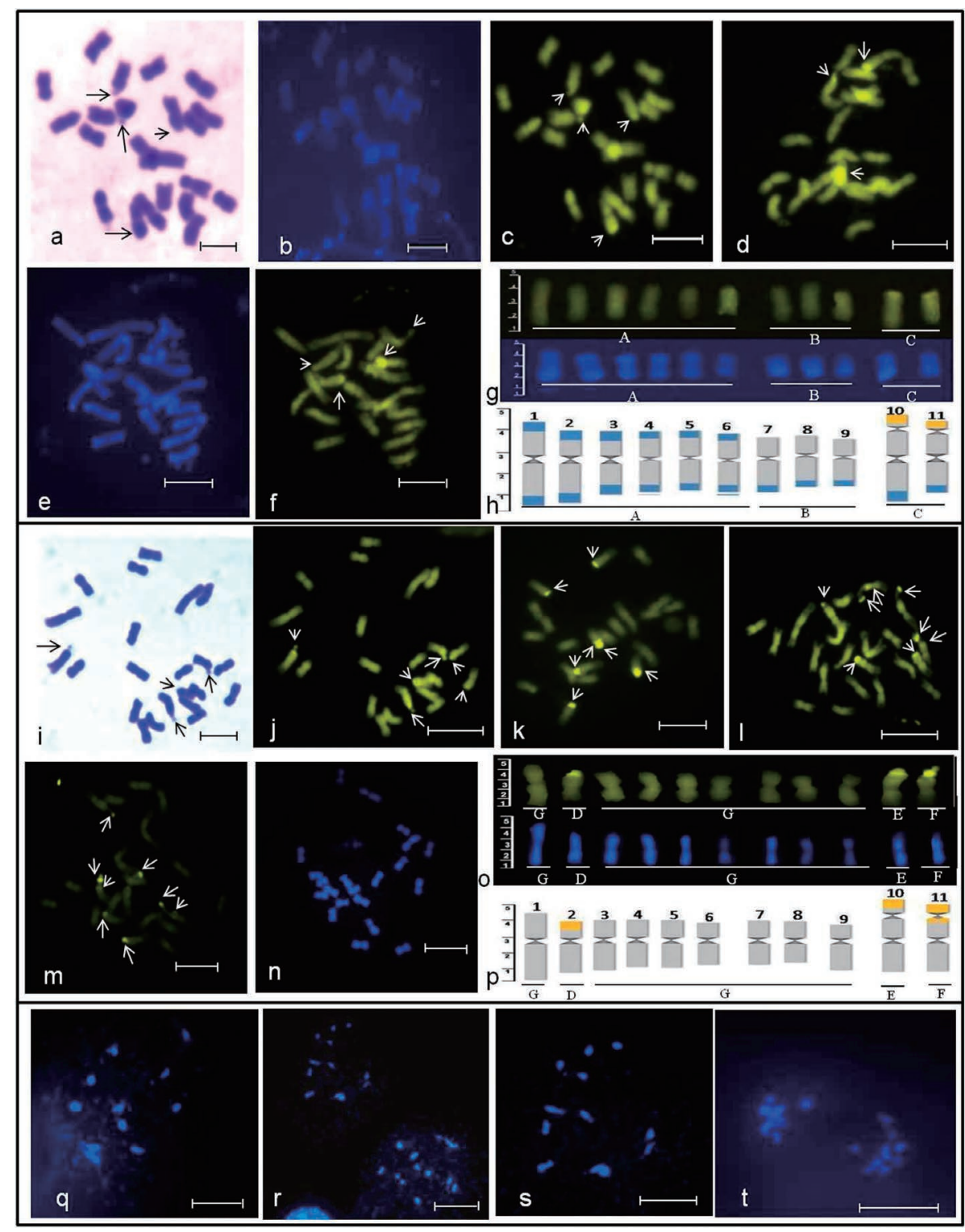

Fig. 3. $\mathrm{a}-\mathrm{f}$ Somatic metaphase chromosomes of TCSF plants stained with Giemsa (a) DAPI (b and e) and CMA (c, d, and f): a-c KOL, d NBP; e-f BEN. g-h Haploid ideogram (g) and karyotype (h) showing CMADAPI banding pattern in the chromosomes of TCSF plants. i-n Somatic chromosomes of TCLF plants stained with Giemsa (i) CMA (j-m) and DAPI (n): i-j BON, k-1 BHA; m-n ADI. o-p Haploid ideogram (o) and karyotype (p) showing CMA banding pattern and lack of DAPI bands in the chromosomes of TCLF plants. Black arrows indicate nucleolar constrictions and white arrows indicate CMA bands. q-t EMA based DAPI stained PMCs showing meiotic chromosomes at metaphase I (q and s) and metaphase II ( $r$ and t) stages: $q-r$ TCSF, $\mathrm{s}-\mathrm{t}$ TCLF. For abbreviation of populations, see Table 1 . Scale bar $=5 \mu \mathrm{m}$.
On the basis of occurrence of DAPI and CMA bands, chromosomes were classified into three distinct types in the TCSF plants such as type A where two distal DAPI bands appeared in the opposite arms of a single chromosome, type B chromosomes having one distal DAPI band and type $\mathrm{C}$ chromosomes with one distal CMA band in the shortest arm and one distal DAPI band in the longest arm. This prototype was found to be consistent in all TCSF plants and hence the DAPI and CMA banding pattern could be determined as $12 \mathrm{~A}+6 \mathrm{~B}+4 \mathrm{C}$ (Fig. $3 \mathrm{~g}-\mathrm{h}$ ). Compared to TCSF, plants of TCLF had chromosomes with different fluorochrome banding patterns namely type D with one distal CMA band, type E with one distal CMA band in the nucleolar chromosomes, type $\mathrm{F}$ with distal CMA band followed by two CMA spots immediately below the second constriction and type $\mathrm{G}$ with no fluorochrome bands. Hence the CMA banding pattern in TCLF plants was determined as $2 \mathrm{D}+2 \mathrm{E}+2 \mathrm{~F}+16 \mathrm{G}$ (Fig. 3o-p).
Meiotic behavior of the plants studied by EMA-DAPI staining technique

Regular chromosome pairing in early prophase I and presence of 11 bivalents in metaphase I confirmed the gametic chromosome number as $n=11$ in TCSF and TCLF plants (Fig. 3q, s). Observation of 11 chromosomes in metaphase II further confirmed regular segregation in both TCSF and TCLF plants (Fig. 3r, t). A chromosomal anomaly in meiotic divisions was not detected.

Status of karyotype evolution determined by PCA and UPGMA analyses

In the principal component analysis, plants of TCLF and TCSF were located at a considerable distance in the component plot. The plot accounted for $96.3 \%$ of the total chromosomal differences between the two types of plants. PC1 accounted for $88.5 \%$ of the total variation whereas PC2 accounted for $7.8 \%$ of the variation 


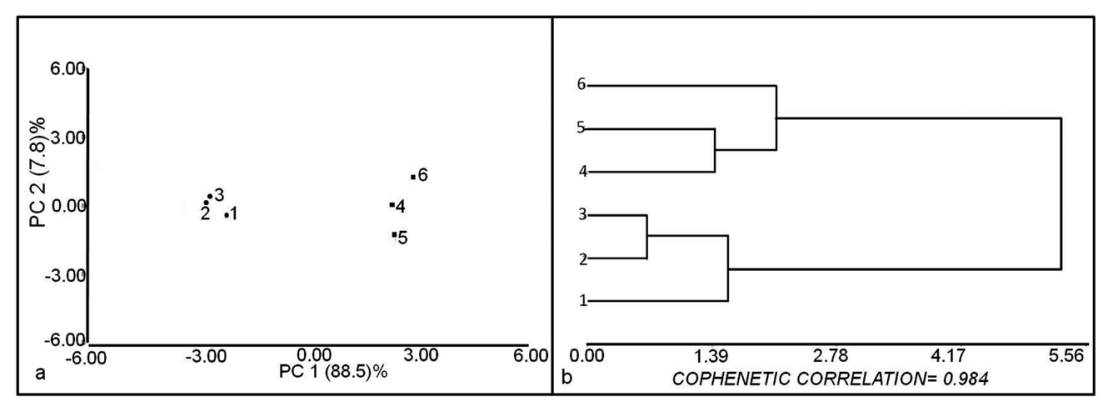

Fig. 4. a Component plot derived after principal component analysis of TCSF $(\mathbf{)})$ and TCLF $(\boldsymbol{\square})$ plants of six populations using karyomorphometric and fluorochrome banding variables, 1: KOL, 2: NBP, 3: BEN, 4: BON, 5: BHA, 6: ADI. b UPGMA dendrogram worked out from average Euclidean distance on the basis of karyomorphometric indices and fluorochrome banding data of TCSF and TCLF plants of six populations, 1: KOL, 2: NBP, 3: BEN, 4: BON, 5: BHA, 6: ADI. For abbreviation of populations, see Table 1.

in the two-dimensional plot (Fig. 4a). PC1 highlighted the variations due to differences in $\mathrm{CV}_{\mathrm{CL}}, \mathrm{CV}_{\mathrm{CI}}, \mathrm{AsK} \%$, DI, $\mathrm{M}_{\mathrm{CA}}$ and number of chromosomes with DAPI bands while PC2 emphasized chromosomal differences owing to variation in $\mathrm{CV}_{\mathrm{r}}$, $\mathrm{AI}$ and number of chromosomes with CMA bands. TCSF and TCLF occupied two spatially distinct groups in the component plot (Fig. 4a). Cluster analysis with quantitative parameters along with CMA and DAPI banding pattern resulted in the formation of two distinct clusters of TCSF and TCLF in the UPGMA dendrogram with a cophenetic correlation of 0.984 which was a good fit between the average linkage matrix and cophenetic value matrix (Fig. 4b).

\section{Discussion}

T. cucumerina was known to have a taxonomic controversy regarding the inclusion of snake gourd as variety since a long time. The naturally growing TCSF was considered recently as the wild type while TCLF (snake gourd) was regarded as the cultivar (Pradheep et al. 2015). We collected populations of both TCSF and TCLF from different regions to see if there is any significant difference in karyotype with respect to geographical variations. The EMA-Giemsa based method of chromosome analysis enabled clarification of chromosome morphology and karyotype analysis in T. cucumerina. Meiotic revision of this species by EMA-DAPI staining was found useful to visualize chromosomes and reconfirm constancy in gametic chromosome number and regular chromosome segregation in both types of plants. Considering the early reports, our study corroborates to the number of chromosomes reported $(2 n=22$, $n=11)$ (McKay 1931, Nakajima 1936, Sen and Datta 1975, Singh and Roy 1975, Datta and Basu 1978, Sarker et al. 1987, Beevy and Kuriachan 1996). TCSF plants of three populations had the same chromosome numbers, similar karyotypes, and banding patterns but had minor differences in a chromosome size range and karyotype asymmetry/symmetry indices. Also, the cultivars had an overall constancy in karyotype attributes among the populations and little difference in chromosome size, pointing towards genomic stability. The difference in karyotypes was mainly pronounced between the TCSF and TCLF in terms of chromosome lengths and centromeric positions which were further reflected in the statistical analysis with karyotype symmetry/asymmetry values. However, the occurrence of three nucleolar chromosome pairs in T. cucumerina and T. anguina was documented in old illustrations (Sarker et al. 1987), while some cultivars of snake gourd were reported to contain variation in chromosome numbers, karyotypes, and a number of satellites chromosomes (Alam et al. 2012, 2018). Presently, four chromosomes with the secondary constriction were clearly observed in both TCSF and TCLF plants, having differences in the centromeric position.

The tendency of having symmetric or asymmetric nature in the karyotype of a species has been appreciated as a standard for cytotaxonomic evaluation (Paszko 2006, Peruzzi and Eroğlu 2013). Inter-chromosomal asymmetry resulting from heterogeneity in chromosome sizes (Levitsky 1931) was prominently acquired in TCLF cultivar. Variations in the relative position of centromeres lead to intra-chromosomal asymmetry (Levitsky 1931) which is also higher in TCLF. Some parameters are inversely related to intra-chromosomal symmetry/asymmetry which had lower values (AI and $\mathrm{TF} \%$ ) in TCLF, supporting higher asymmetry in its karyotype. Parameters like AI (Paszko 2006) combines the impact of inter- and intra-chromosomal variations and were also indicative of higher asymmetry in TCLF. The data are based on five TCSF and five TCLF plants from each population and hence the overall asymmetric inclination of karyotype in TCLF is statistically significant.

CMA and DAPI stainings accentuated dissimilarities in chromosome architectures. The presence of DAPI bands in all chromosomes of TCSF (types A, B, and $\mathrm{C}$ ) demarcated it from TCLF which lacked such bands altogether. The occurrence of DAPI bands in all chromosomes was previously documented in $T$. dioica 
(Bhowmick and Jha 2015). There might be a tendency for acquiring DAPI bands in the genus which can be confirmed after study in other species of Trichosanthes. The presence of four distal CMA bands in TCSF plants differentiated them from TCLF plants with six distal CMA bands. Previously, CMA bands have been suggested to be GC-rich sequences corresponding to NORs (Schweizer 1976, Guerra 2000) which in this case was corroborating with four nucleolar chromosomes of both TCSF (type C) and TCLF (types E and F) with distal CMA bands. Interestingly, the type F nucleolar chromosome pair of TCLF had an additional interstitial (immediately below the secondary constriction) band. Again, the type D non-nucleolar chromosomes with distal CMA bands were another remarkable feature in TCLF plants. However, the particular nature of these non-nucleolar or interstitial CMA bands awaits the application of in situ hybridization with rDNA probe.

Fluorochrome banding patterns and quantitative karyomorphometry parameters directly related to karyotype asymmetry were utilized for assessing the statistical difference between the two types of plants. Inversely related parameters (TF\% and Syi) and qualitative indices like Stebbins category were ruled out. The UPGMA analysis and PCA yielded distinct grouping of TCSF plants apart from TCLF and emphasized delineation of the two. The evolution of cultivars might come about as a consequence of artificial selection in agricultural practice. The mechanism of whether and how these processes may lead to certain chromosomal changes is not known in this case. The cultivar 'Anguina' share same chromosome numbers with TCSF unlike many cultivated cucurbits with higher ploidy (Waminal and Kim 2015). A detailed investigation of chromosome morphology and fluorochrome banding pattern clarified the difference between the T. cucumerina and its cultivar 'Anguina.' In accordance with the morphological basis of delineation, karyotype and fluorochrome banding pattern analysis in our study provides an archetype to complement further cytotaxonomic revision of $T$. cucumerina. These two types of plants have many similarities in morphological features other than fruit because of which snake gourd was regarded as the cultivar and not a distinct species or subspecies (Pradheep et al. 2015). Since TCSF and TCLF plants have the same ploidy but the difference in karyotype attributes with respect to the difference in fruit morphology, we are far from making any conclusion to resolve the taxonomic status of these plants. Karyotype differences were previously documented between the varieties of bitter gourd (Ghosh et al. 2018) and varieties and cultivars of cucumber (Zhao et al. 2011, Zhang et al. 2012). It would be interesting to see the extent of phylogenetic divergence between TCSF and TCLF in future genome analysis. Results of our cytogenetic study lay a foundation for such investigations in T. cucumerina that might also facilitate selective breeding among plants with desirable agricultural and medicinal qualities.

\section{Acknowledgements}

SJ is thankful to the National Academy of Sciences (NASI, Allahabad, India), for the award of NASI Senior Scientist Fellowship and providing the financial support to continue the research. The authors wish to express their sincere thanks to the Head, Department of Botany and Programme Coordinator, CAS in Botany, University of Calcutta for providing the facilities to carry out this research.

\section{References}

Alam, M. M., Haque, S. M. and Ghosh, B. 2018. Karyomorphological studies of six commercially cultivated edible cucurbits: Bitter gourd, sponge gourd, ridge gourd, snake gourd, ash gourd and cucumber. Caryologia 71: 150-159.

Alam, S. S., Jahan, N., Habib, M. A. and Islam, M. N. 2012. Cytogenetical and molecular characterization of five commercial varieties in Trichosanthes anguina L. Cytologia 77: 155-162.

Albert, P. S., Gao, Z., Danilova, T. V. and Birchler, J. A. 2010. Diversity of chromosomal karyotypes in maize and its relatives. Cytogenet. Genome Res. 129: 6-16.

Ali, A. M. and Al-Hemaid, F. M. A. 2010. Trichosanthes anguina L. is variety of Trichosanthes cucumerina L. - evidence based on molecular phylogenetic analysis of internal transcribed spacer (ITS) sequences of nuclear ribosomal DNA. Int. J. Mol. Biol. 1: $1-14$.

Arano, H. 1963. Cytological studies in subfamily Carduoideae (Compositae) of Japan. IX. Bot. Mag. Tokyo 76: 32-39.

Beevy, S. S. and Kuriachan, P. 1996. Chromosome numbers of south Indian Cucurbitaceae and a note on the cytological evolution in the family. J. Cytol. Genet. 31: 65-71.

Bhowmick, B. K. and Jha, S. 2015. Differential heterochromatin distribution, flow cytometric genome size and meiotic behavior of chromosomes in three Cucurbitaceae species. Sci. Hortic. 193: 322-329.

Bhowmick, B. K., Jha, T. B. and Jha, S. 2012. Chromosome analysis in the dioecious cucurbit Coccinia grandis (L.). Voigt. Chromosome Sci. 15: 9-15.

Chakravarty, H. L. 1959. Monograph of Indian Cucurbitaceae (taxonomy and distribution). Rec. Bot. Surv. India 17: 1-234.

Chakravarty, H. L. 1982. Cucurbitaceae. In: Jain, S. K. (ed.). Fascicles of Flora of India 11. Bot. Surv. India, Calcutta. pp. 1-136.

Cooper, W. E. and de Boer, H. J. 2011. A taxonomic revision of Trichosanthes L. (Cucurbitaceae) in Australia, including one new species from the Northern Territory. Austrobaileya 8: 364-386.

Datta, S. K. and Basu, R. K. 1978. Cytomorphological, biochemical and palynological studies in Trichosanthes anguina L. and $T$. cucumerina L. Cytologia 43: 107-117.

Fukui, K. 1996. Plant Chromosomes at Mitosis. In: Fukui, K. and Nakayama, S. (eds.). Plant Chromosomes: Laboratory Methods. CRC Press Inc., Boca Raton. pp. 1-17.

Ghosh, I., Bhowmick, B. K. and Jha, S. 2018. Cytogenetics of two Indian varieties of Momordica charantia L. (bittergourd). Sci. Hortic. 240: 333-343.

Greilhuber, J. and Speta, F. 1976. C-banded karyotypes in the Scilla hohenackeri group, S. persica, and Puschkinia (Liliaceae). Plant Syst. Evol. 126: 149-188.

Guerra, M. 2000. Patterns of heterochromatin distribution in plant 
chromosomes. Genet. Mol. Biol. 23: 1029-1041.

Haines, H. H. 1922. The Botany of Bihar and Orissa. Adlard \& Son Co. Ltd., London.

Huziwara, Y. 1962. Karyotype analysis in some genera of Compositae. VIII. Further studies on the chromosomes of Aster. Am. J. Bot. 49: 116-119.

Icso, D., Molnar-Lang, M. and Linc, G. 2014. Constructing an alternative wheat karyotype using barley genomic DNA. J. Appl. Genet. 56: $45-48$.

Jeffrey, C. 1980a. A review of the Cucurbitaceae. Bot. J. Linn. Soc. 81: 233-247.

Jeffrey, C. 1980b. Further notes on Cucurbitaceae: V: The Cucurbitaceae of the Indian subcontinent. Kew Bull. 34: 789-809.

Lavania, U. C. and Srivastava, S. 1992. A simple parameter of dispersion index that serves as a adjunct to karyotype asymmetry. J. Biosci. 17: 179-182.

Levan, A., Fredga, K. and Sandberg, A. A. 1964. Nomenclature for centromeric position on chromosomes. Hereditas 52: 201-220.

Levitsky, G. A. 1931. The karyotype in systematics. Bull. Appl. Bot. Genet. Plant Breed. 27: 220-240.

Liang, G. and Chen, H. 2015. Scaling chromosomes for an evolutionary karyotype: A chromosomal tradeoff between size and number across woody species. PLoS One 10: e0144669.

Linnaeus, C. 1753. Species Plantarum 2: 1008.

Marghali, S., Zitouna, N., Gharbi, M., Chennaoui-Kourda, H. and Trifi-Farah, N. 2014. Morphological and molecular characters: Congruence or conflict in the phylogeny of Sulla species? Austr. J. Crop Sci. 8: 148-158.

McKay, J. W. 1931. Chromosome studies in the Cucurbitaceae. Univ. Calif. Berkeley Publ. Bot. 16: 339-350.

McVean, G. 2009. A genealogical interpretation of principal components analysis. PLoS Genet. 5: e1000686.

Nakajima, G. 1936. Chromosome numbers in some crops and wild angiosperms. Jpn. J. Genet. 12: 211-217.

Paszko, B. 2006. A critical review and a new proposal of karyotype asymmetry indices. Plant Syst. Evol. 258: 39-48.

Peruzzi, L. and Eroğlu, H. E. 2013. Karyotype asymmetry: Again, how to measure and what to measure? Comp. Cytogenet. 7: 1-9.

Pradheep, K., Pani, D. R. and Bhatt, K. C. 2015. Taxonomic notes on the Trichosanthes cucumerina group (Cucurbitaceae) from India.
Novon 24: 39-46.

Renner, S. S. and Pandey, A. K. 2013. The Cucurbitaceae of India: Accepted names, synonyms, geographic distribution and information on images and DNA sequences. PhytoKeys 20: 53-118.

Sarker, D. D., Datta, K. B. and Sen, R. 1987. Cytomorphology of some wild and cultivated members of Trichosanthes L. Cytologia 52: 405-417.

Schaefer, H. and Renner, S. S. 2011. Phylogenetic relationships in the order Cucurbitales and a new classification of the gourd family (Cucurbitaceae). Taxon 60: 122-138.

Schweizer, D. 1976. Reverse fluorescent chromosome banding with chromomycin and DAPI. Chromosoma 58: 307-324.

Seijo, J. G. and Fernández, A. 2003. Karyotype analysis and chromosome evolution in South American species of Lathyrus (Leguminosae). Am. J. Bot. 90: 980-987.

Sen, R. and Datta, K. B. 1975. Cytogenetic studies in cultivated Trichosanthes L. (Cucurbitaceae) and their wild relatives. Proc. Indian Sci. Congr. Soc. 10: 62.

Singh, A. K. and Roy, R. P. 1975. Cytomorphological studies in polyploids of Trichosanthes anguina L. Cytologia 40: 13-20.

Waminal, N. E. and Kim, H. H. 2015. FISH karyotype analysis of four wild Cucurbitaceae species using $5 \mathrm{~S}$ and $45 \mathrm{~S}$ rDNA probes and the emergence of new polyploids in Trichosanthes kirilowii Maxim. Korean J. Hortic. Sci. Technol. 33: 869-876.

Watanabe, K., Yahara, T., Denda, T. and Kosuge, K. 1999. Chromosomal evolution in the genus Brachyscome (Asteraceae, Astereae): Statistical tests regarding correlation between changes in karyotype and habit using phylogenetic information. J. Plant Res. 112: $145-161$.

Zarco, C. R. 1986. A new method for estimating karyotype asymmetry. Taxon 35: 526-530.

Zhang, C., Kikuchi, S. and Koba, T. 2012. Karyotype comparison of Indian and Japanese cucumber cultivars by fluorescence in situ hybridization probed with tandem repeat sequences. Chromosome Sci. 15: 17-21.

Zhao, X., Lu, J., Zhang, Z., Hu, J., Huang, S. and Jin, W. W. 2011. Comparison of the distribution of the repetitive DNA sequences in three variants of Cucumis sativus reveals their phylogenetic relationships. J. Genet. Genomics 38: 39-45. 